\title{
Spontaneous rupture of the non-gravid uterus
}

\author{
Leong Chee Weng, ${ }^{1}$ Tulsi Menon, ${ }^{2}$ Graham Hool ${ }^{2}$
}

${ }^{1}$ Royal Perth Hospital, Perth, Western Australia, Australia ${ }^{2}$ Department of General Surgery, Royal Perth Hospital, Perth, Western Australia, Australia

Correspondence to Dr Leong Chee Weng, Dleong87@gmail.com
To cite: Weng $\mathrm{LC}$, Menon T, Hool G. BMJ Case Rep Published online: [please include Day Month Year] doi:10.1136/bcr-2013008895

\section{SUMMARY}

A 71-year-old woman presents with acute onset abdominal pain with sepsis. Initial workup with an abdominal CT scanning revealed intraperitoneal air with an enlarged uterus and thickened small bowel loops. Haemodynamic instability with sepsis prompted urgent laparotomy. At laparotomy, spontaneous perforation of the uterus was identified. The cause was due to infarction of the myometrium. This occurred on a background of not having any previous instrumentation of the uterus.

\section{BACKGROUND}

- Spontaneous uterine rupture is rare but should be a differential in a woman with acute onset abdominal pain.

- There are very few cases published. This particular case highlights infarction of the myometrium as a cause which has not been previously described.

\section{CASE PRESENTATION}

A 71-year-old woman presented to the emergency department with a $24 \mathrm{~h}$ history of sudden onset of severe generalised abdominal pain. The pain was constant in nature with associated rigors, hypotension and vomiting. She reports feeling generally unwell for the preceding week prior to presentation. Systems review was unremarkable. Her medical history included hypertension, hyperlipidaemia, heavy smoking, diabetes mellitus, stroke with right hemiparesis and previous open cholecystectomy. She had four children all of whom were delivered via spontaneous delivery. There was no history of previous uterine or cervical instrumentation. She did not have any evidence of sexually transmitted diseases. On presentation, she was hypotensive 82/54, tachycardic at 104 and febrile with a generally guarded abdomen with peritonitis.

\section{INVESTIGATIONS}

Plain erect chest radiograph was unremarkable. Laboratory investigations revealed a haemoglobin of $10.1 \mathrm{~g} / 100 \mathrm{ml}$ with a leucocytes of $15000 / \mathrm{mm}^{3}$. Blood cultures were sent which returned negative. Renal, liver and clotting function tests were normal. In the context of severe abdominal tenderness an urgent CT scan of the abdomen was performed which showed thickened small bowel loops and gross distension of the uterus with fluid in the pelvis (figure 1).

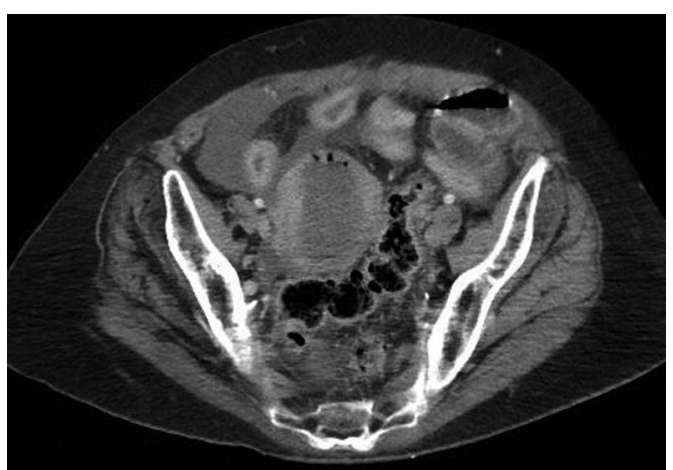

Figure 1 CT scan of dilated uterus with pus.

\section{DIFFERENTIAL DIAGNOSIS}

Differential diagnosis included perforated bowel, complicated diverticulitis, visceral ischaemia and peptic ulcer perforation.

\section{TREATMENT}

She was initially resuscitated with 2 litres of crystalloid with good response, her blood pressure increasing to $115 / 75$. Intravenous antibiotics, ceftriaxone and metronidazole were given. An urgent laparotomy and hysterectomy were subsequently performed. There was evidence of $600 \mathrm{ml}$ of pus and a perforated uterus with approximately $100 \mathrm{ml}$ of intraperitoneal blood without any other intra-abdominal cause for her presentation. The abdominal fluid was sent for microscopy, culture and sensitivity, which grew proteus mirabilis susceptible to ceftriaxone, norfloxacin and piperacillin with tazobactam. Antibiotics were given for a total course of 2 weeks postoperatively.

\section{OUTCOME AND FOLLOW-UP}

The postoperative course was complicated by a severe systemic inflammatory response with multiple organ failure. After a prolonged intensive care unit stay requiring inotropic support, prolonged ventilation and continuous venovenous haemodiafiltration (CVVHDF), she made full recovery and was discharged 46 days postoperatively. Further questioning revealed that her mother had also suffered from spontaneous uterine rupture at a similar age. Histopathology results revealed atherosclerosis of the myometrial vessels and infarction as the cause of spontaneous perforation.

\section{DISCUSSION}

Spontaneous rupture of the uterus is a rare but documented finding in pregnant women. The occurrence of spontaneous uterine rupture in a non-gravid woman is much rarer. The aetiology is unclear; however, multiple factors have been 
attributed to its occurrence. These factors include leiomyoma, infections and carcinoma. ${ }^{1-3}$

Only a few cases of spontaneous rupture of the uterus in a non-gravid woman without trauma or uterine anomaly have been described previously. The first patient had multiple prior abdominal surgeries and deep cauterisation of her cervix as well as oestrogen withdrawal bleeding. ${ }^{4}$ The cauterisation of her cervix had resulted in cervical stenosis with withdrawal bleeding causing increasing uterine pressure and subsequent rupture. The second patient had fulminant pelvic infection ${ }^{5}$ prior to the rupture. The third case reported that the patient had a dilation and curettage a few months prior. ${ }^{6}$ The dilatation and curettage resulted in a focal myometrial weakness predisposing it to spontaneously rupturing.

The cause of perforation in this case on histopathology was reported as myometrial ischaemia with evidence of atherosclerotic vessels. There was full thickness necrosis with neutrophil infiltration. There was no evidence of cervical stenosis or anatomical defects. This is consistent with her vascular risk factors including hypertension, hyperlipidaemia, diabetes mellitus, being a heavy smoker with an 80-pack-year history of smoking and a previous stroke. The ischaemia of the myometrium resulted in necrosis, a nidus for infection and abscess formation with resulting sepsis. The initial infarction of the uterine wall and subsequent inflammation and infection consistent with her history of feeling unwell for a week prior to presentation with sepsis resulted in the perforation.

The diagnosis of spontaneous uterine rupture in a non-gravid woman is very difficult. The classical symptoms described are abdominal pain with shock and vaginal bleeding. Abdominal pain is reported to occur in up to $13-60 \%$ of cases, vaginal bleeding in 11-67\% of patients and haemorrhagic shock in up to $46 \%$ of patients. ${ }^{45}$ The management of spontaneous uterine rupture is surgical. These surgical options include primary repair, where the myometrium is directly oversewn. ${ }^{6}$ Other cases including our patient underwent hysterectomy with good postoperative outcomes reported. ${ }^{4-7}$

While rare, the diagnosis of spontaneous uterine rupture should be considered in the presentation of any woman with abdominal pain. The cause for spontaneous rupture in this patient was found to be secondary to ischaemia with an underlying vascular disease.

\section{Learning points}

- Spontaneous rupture of the uterus is a differential in any non-gravid woman presenting with acute abdominal pain.

- The causes of spontaneous rupture of the non-gravid uterus include ischaemia, distal cervical stenosis with hormonal therapy, prior instrumentation and fulminant pelvic infection.

- Spontaneous uterine rupture can be managed with primary repair or hysterectomy.

Contributors LCW is responsible for the literature review and write-up of the case with the guidance and review of TM and GH who are both consultants in general surgery.

Competing interests None.

Patient consent Obtained.

Provenance and peer review Not commissioned; externally peer reviewed.

\section{REFERENCES}

1 Eden RD, Parker RT, Gall SG. Rupture of the pregnant uterus: a 53-year review. Obstet Gynecol 1986;68:671-4.

2 Kieser KE, Baskett TF. A 10-year population-based study of uterine rupture. Obstet Gynecol 2002;100:749-53.

3 Golan A, Sandbank 0, Rubin A. Rupture of the pregnant uterus. Obstet Gynecol 1980;56:549-54.

4 Frech HC. Spontaneous rupture of a non-gravid uterus. South Med J 1949:42:1088.

5 Bornstien FP. Spontaneous rupture of the nonpregnant uterus. Postgrad Med 1952;12:534-6.

6 Herrera FA, Haanein AH, Bansal V. Atraumatic spontaneous rupture of the non-gravid uterus. J Emerg Trauma Shock 2011;4:439.

7 Mokgokong ET, Marivate M. Treatment of the ruptured uterus. S Afr Med J 1976;50:1621-4.

\footnotetext{
Copyright 2013 BMJ Publishing Group. All rights reserved. For permission to reuse any of this content visit http://group.bmj.com/group/rights-licensing/permissions.

BMJ Case Report Fellows may re-use this article for personal use and teaching without any further permission.

Become a Fellow of BMJ Case Reports today and you can:

- Submit as many cases as you like

- Enjoy fast sympathetic peer review and rapid publication of accepted articles

- Access all the published articles

- Re-use any of the published material for personal use and teaching without further permission

For information on Institutional Fellowships contact consortiasales@bmjgroup.com

Visit casereports.bmj.com for more articles like this and to become a Fellow
} 